TITLE:

\title{
Nonlinear Optical Phenomena Induced by Intense Single-Cycle Terahertz Pulses
}

\author{
$\operatorname{AUTHOR}(S)$ :
}

Hirori, Hideki; Tanaka, Koichiro

\section{CITATION:}

Hirori, Hideki ... [et al]. Nonlinear Optical Phenomena Induced by Intense Single-Cycle Terahertz Pulses. IEEE Journal of Selected Topics in Quantum Electronics 2013, 19(1): 8401110.

\section{ISSUE DATE:}

2013-01

URL:

http://hdl.handle.net/2433/169793

\section{RIGHT:}

(c) 2012 IEEE. Personal use of this material is permitted. Permission from IEEE must be obtained for all other uses, in any current or future media, including reprinting/republishing this material for advertising or promotional purposes, creating new collective works, for resale or redistribution to servers or lists, or reuse of any copyrighted component of this work in other works.; この論文は出版社版でありません。引用の際には出版社版をご確認ご利用ください。; This is not the published version. Please cite only the published version. 


\title{
Nonlinear Optical Phenomena Induced by Intense Single-Cycle Terahertz Pulses
}

\author{
Hideki Hirori $^{1,2, *}$ and Koichiro Tanaka ${ }^{1,2,3}$ \\ ${ }^{1}$ Institute for Integrated Cell-Material Sciences, Kyoto University, Sakyo-ku, Kyoto 606-8501, Japan \\ ${ }^{2}$ Core Research for Evolutional Science and Technology, Japan Science and Technology Agency, \\ Kawaguchi-shi, Saitama 332-0012, Japan \\ ${ }^{3}$ Department of Physics, Graduate School of Science, Kyoto University, Sakyo-ku, Kyoto 606-8502, Japan
}

\begin{abstract}
Recent developments in high-power table-top terahertz (THz) pulse sources enable us to coherently drive low-energy transitions into the nonlinear regime and to study fascinating nonlinear effects in various materials. This review article describes a tilted-pulse-front pumping scheme with a $\mathrm{LiNbO}_{3}$ crystal to generate intense single-cycle THz pulses. This scheme is capable of generating intense singlecycle THz pulses induce strong spectral modulations in the excitonic and band-edge absorption of ZnSe/ZnMgSSe multiple quantum wells (MQW). Furthermore, it is shown that a $1 \mathrm{MV} / \mathrm{cm}$ electric field of a THz pulse allows us to excite electrons from the valence to conduction band of GaAs/AIGaAs MQW and observe a bright exciton luminescence.
\end{abstract}

Index Terms - Electroluminescent devices, Nonlinear optics, Semiconductor materials, Terahertz pulse generation, Ultrafast optics

\section{INTRODUCTION}

$\mathrm{T}$ he terahertz $(\mathrm{THz})$ region of the electromagnetic spectrum, i.e., usually defined as spanning two decades from 0.1 to $10 \mathrm{THz}$ or 3 to $300 \mathrm{~cm}^{-1}$, is of critical importance in the investigation of matter in physical, chemical, and biological systems. This frequency region is also known as the far-infrared range and contains spectroscopic signatures for various energy modes, e.g., density oscillations of carrier plasmas, superconducting energy gaps, internal transition energies of excitons in semiconductors, spin waves, weakly bonded molecular crystals, phonons in crystalline solids, and hydrated biological matter. However, because of the lack of suitable light sources and detectors in the $\mathrm{THz}$ spectral region, only rather indirect investigation of these low-energy modes by using near-infrared to visible light pulses has been possible.

During the past two decades, the advent and development of efficient $\mathrm{THz}$ pulse sources relying on the frequency conversion of femtosecond lasers have enabled time-domain spectroscopy (TDS) [1-5]. This technique makes use of subpicosecond pulses of freely propagating electromagnetic radiation in the $\mathrm{THz}$ range and enables characterizing of the complete electric field of a THz pulse to yield full phase and amplitude information by using photoconductive [6-9] and free-space electro-optic sampling (EOS) [10-14]. The complex dielectric function of a sample in the beam path can thus be determined directly without having to rely on Kramers-Kronig relations. This is an advantage over typical detectors that measure only intensity, e.g., liquid-helium cooled bolometers or pyroelectric detectors, and it can also yield very high signal-to-noise ratio measurements because of its insensitivity to the blackbody radiation of the environment. The two main methods of generating $\mathrm{THz}$ pulses with femtosecond lasers were established early on and are still the most widely used: photoconductive switches $[8,15,16]$ and optical rectification

Manuscript received April 5, 2012 (date on which paper was submitted for review). Corresponding author: H. H. (e-mail: hirori@icems.kyotou.ac.jp).

Digital Object Identifier inserted by IEEE
(OR) [10]. Initially, the available frequency spectrum that could be generated and detected in the time domain was limited to a few THz. Advances in the generation and detection of broadband $\mathrm{THz}$ pulses have pushed this limit into the midinfrared and recently into the near-infrared, reaching frequencies beyond $100 \mathrm{THz}(\lambda=3 \mu \mathrm{m})$ [17-21].

Accordingly, owing to the development of sources and detectors of coherent $\mathrm{THz}$ pulses, much progress has been made in the past decades in understanding the physics of elementary electronic excitations. TDS not only allows for characterization of various energy modes under steady-state conditions; it is also inherently suited for nonequilibrium measurements: an optical pulse can be used to excite charge carriers and the subsequent evolution of these carriers can be monitored with a time resolution on the order of tens of femtoseconds. [22-30].

Moreover, laser-based high-power $\mathrm{THz}$ pulse sources in the microjoule range were recently developed and their strong electric fields can be used to excite samples that have resonances or other spectral features in the $\mathrm{THz}$ frequency range [31-34]. In addition, table-top $\mathrm{THz}$ sources have many promising applications such as in large-scale object imaging, medical diagnosis and treatment, and remote sensing for security purposes [35-37]. Very high average power continuous wave (cw) sources such as nanosecond gas lasers and free-electron lasers have even enabled studies of nonlinear effects in the THz range [38-43]. Moreover, laser-based strong $\mathrm{THz}$ radiation at frequencies of $20-80 \mathrm{THz}$ can be generated by difference frequency mixing in GaSe crystals [44-46], and peak electric fields of up to $100 \mathrm{MV} / \mathrm{cm}$ can be obtained in a multi-oscillation cycle regime centered around $40 \mathrm{THz}$ [45], and up to $10 \mathrm{MV} / \mathrm{cm}$ in a single-oscillation cycle regime centered around $20 \mathrm{THz}$ [46].

Nonetheless, the generation of high-power $\mathrm{THz}$ single-cycle pulses above $1 \mathrm{MV} / \mathrm{cm}$ in the range of $0.1-10 \mathrm{THz}$, the socalled $\mathrm{THz}$ gap, is still challenging, due to the limited availability of appropriate $\mathrm{THz}$ emitters. Only recently have 1 $\mathrm{MV} / \mathrm{cm}$ single-cycle pulses at a $0.8 \mathrm{THz}$ central frequency 
been demonstrated by $\mathrm{OR}$ in $\mathrm{LiNbO}_{3}$ by using the tilted pulse front technique (which will be discussed in this review paper). $[33,34,47,48]$. Other laser-based approaches like laser-plasmabased techniques have led to the generation of THz-pulse electric fields as high as a hundred of $\mathrm{kV} / \mathrm{cm}$, with bandwidths up to $75 \mathrm{THz}$ [49-53]. THz pulse generation in plasmas, unlike typical nonlinear crystal-based techniques, does not suffer from a damage threshold for the emitter or material absorptions such as optical phonons limiting the available bandwidth of the pulses. Conversely, THz pulses can also be detected in gases by using THz-induced second-harmonic generation (SHG) or fluorescence [54-58]. Organic nonlinear crystals called DAST have also been proposed as efficient table-top $\mathrm{THz}$ emitters. $\mathrm{THz}$ pulses at a central frequency of $2.1 \mathrm{THz}$ with a $1 \mathrm{MV} / \mathrm{cm}$ electric field strength can be generated by OR in a crystal pumped at the signal wavelength of a powerful optical parametric amplifier utilizing a multi-mJ Ti:sapphire laser system operating at $100 \mathrm{~Hz}$ [59].

The remainder of this paper details a tilted-pump-pulsefront scheme (TPFP) using a $\mathrm{LiNbO}_{3}(\mathrm{LN})$ crystal to generate intense single-cycle $\mathrm{THz}$ pulses [48]. It shows that intense single-cycle THz pulses induce strong spectral modulations in the excitonic and band-edge absorptions of $\mathrm{ZnSe} / \mathrm{ZnMgSSe}$

(a)

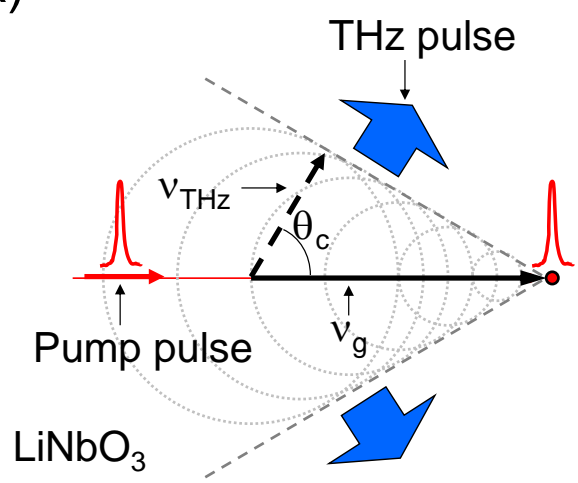

(b)

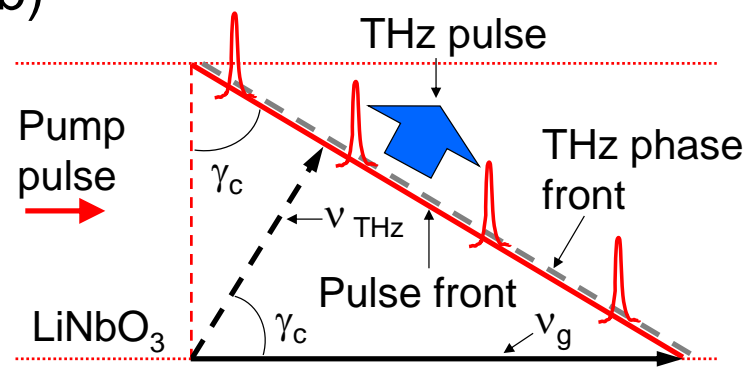

Fig. 1. (a) For the Cherenkov geometry, the THz radiation is emitted as a cone due to OR of a subpicosecond optical pulse moving at a velocity $v_{\mathrm{g}}\left(\omega_{0}\right)$, which is greater than the $\mathrm{THz}$ phase velocity $v_{\mathrm{THz}}$ of the emitted radiation. (b) Velocity matching using tilted-pulse-front pumping (TPFP) scheme creating a plane $\mathrm{THz}$ wave. The thick red/solid line indicates the pump pulse front, the thick grey/dashed line indicates the $\mathrm{THz}$ phase front. The arrows indicate the propagations direction and velocities of these two surfaces. Figure adapted from Ref. [70]. multiple quantum wells (MQW) [60]. Furthermore, it is indicated that a $1 \mathrm{MV} / \mathrm{cm}$ electric field of a $\mathrm{THz}$ pulse allows us to excite electrons from the valence band to the conduction band of GaAs/AlGaAs MQW and observe exciton luminescence [61].

\section{InTENSE Terahertz Pulse Generation}

In 2002, Hebling et al. proposed a tilted-pulse-front pumping scheme for efficient phase-matched $\mathrm{THz}$ pulse generation using stochiometric $\mathrm{LiNbO}_{3}(\mathrm{LN})$ crystals [47]. Since then, LN has become a widely used material for highpower $\mathrm{THz}$ generation with OR $[33,34,48,62-70]$. In addition to its large effective nonlinearity, LN has a bandgap much larger than that of semiconductors that allows only threephoton absorptions to be effectively used at a pump wavelength of $800 \mathrm{~nm}$. This allows for high pump intensities, essential for high-energy $\mathrm{THz}$ pulse generation. Nonetheless, the $\mathrm{THz}$ refractive index of $\mathrm{LN}$, at around 5, is significantly larger than the optical group index at around 2, and therefore, no collinear velocity matching is possible. In bulk LN, singlecycle $\mathrm{THz}$ pulses with high conversion efficiency can be generated by using the tilted-pulse-front pumping with noncollinear velocity matching, as described in the following section.

\section{1) Cherenkov radiation from femtosecond optical} pulses in $\mathrm{LiNbO}_{3}$ crystal

In the Cherenkov geometry (see Fig. 1(a)), which was first used for $\mathrm{THz}$ generation by optical rectification (OR) $[10,71,72], \mathrm{THz}$ radiation from bulk $\mathrm{LN}$ or $\mathrm{LiTaO}_{3}$ is generated by OR of a femtosecond near-infrared (NIR) pump pulse with a tight point or line focus [73,74]. The generated $\mathrm{THz}$ radiation propagates along a cone with an angle $\theta_{\mathrm{c}}$ determined by

$$
\theta_{c}=\cos ^{-1}\left(\frac{v_{T H z}^{p h}}{v_{N I R}^{g}}\right)=\cos ^{-1}\left(\frac{n_{N I R}^{g}}{n_{T H z}}\right),
$$

where $v_{T H z}^{p h}$ and $v_{N I R}^{g}$ are respectively the phase velocity of the generated $\mathrm{THz}$ radiation and the group velocity of the pump pulse, and $n_{T H z}$ and $n_{N I R}^{g}$ are respectively the refractive index in the THz range and the group index of the NIR pump pulse. Because the refractive index in the $\mathrm{THz}$ range is more than two times larger than the one in the visible, the angle of the $\mathrm{THz}$ radiation will be as large as around $60^{\circ}$. Such a propagation characteristic makes it very difficult to collect $\mathrm{THz}$ radiation for applications.

In particular, as for any nonlinear optical frequency conversion process involving a long interaction length, for OR a phase-matching condition between the frequency components involved has to be fulfilled in order to obtain the largest conversion efficiency. Specifically, for collinear geometry the phase-matching condition requires the group velocity of the pump pulse to be equal to the phase velocity of the generated $\mathrm{THz}$ radiation [5]: 


$$
v_{N I R}^{g}=v_{T H z}^{p h}
$$

This should further require the equality of the corresponding indices:

$$
n_{N I R}^{g}=n_{T H z}^{p h}
$$

It is obvious that the large difference of refractive indices makes velocity matching impossible in such important wide bandgap dielectric materials as LN.

\section{2) Principle and realization of tilted-pulse-front excitation}

In the tilted-pulse-front pumping (TPFP), the $\mathrm{THz}$ radiation generated by the tilted pulse front of the pump propagates perpendicularly to this front (Fig. 1(b)) with the $\mathrm{THz}$ phase velocity $v_{T H z}^{p h}$, according to Huygens' principle. This leads to a noncollinear propagation geometry, where the angle between the propagation directions of the $\mathrm{THz}$ radiation and the pump pulse is equal to the tilt angle $\gamma_{\mathrm{c}}$ of the pump pulse front relative to the pump phase fronts (which is perpendicular to the pump propagation direction). The $\mathrm{THz}$ wave propagates with a fixed phase relative to the pump pulse front if the following velocity-matching condition is fulfilled:

$$
v_{N I R}^{g} \cos \gamma_{c}=v_{T H z}^{p h} .
$$

\section{(a)}

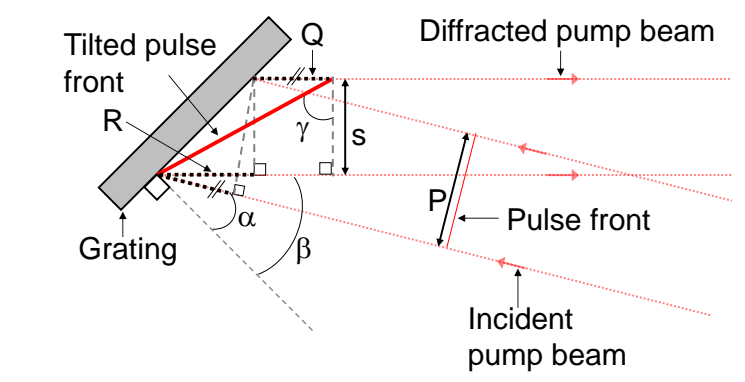

(b)

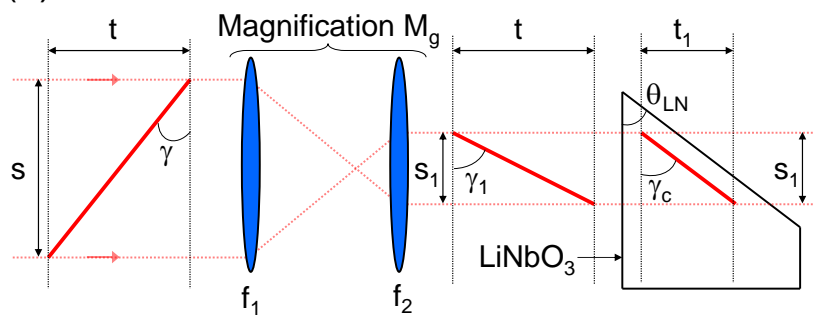

Fig. 2. To the calculation of the tilt angle $\gamma_{c}$ of pump pulse front in $\mathrm{LiNbO}_{3}$ crystal. (a) Diffraction of a single wavelength component of an optical pulse beam incident on a grating at an oblique angle of incidence. The thick red/solid line indicates the pump pulse front. (b) After the grating shown in (a), further tilting of the pump pulse front with lens pair (a magnification of $M_{\mathrm{g}}$ ) and in $\mathrm{LiNbO}_{3}$ crystal.
In the case of LN where $v_{N I R}^{g}>>v_{T H z}^{p h}$, or equivalently $n_{N I R}^{g}<<n_{T H z}^{p h}$, equation (4) can be fulfilled by choosing an appropriate pulse-front-tilt angle $\gamma_{\mathrm{c}}$. To achieve noncollinear velocity matching, a tilt angle as large as around $60^{\circ}$ is necessary inside the LN crystal because $n_{T H_{z}}^{p h}(\sim 5)$ is much higher than $n_{N I R}^{g}(\sim 2)$ [66]. Such a large angle can be realized by using a grating and two cylindrical lenses, as shown in Figs. 2(a) and (b).

Consider the pulse front of the pump beam of a "single wavelength" incident on a diffraction grating at an angle $\alpha$ and diffracted at an angle $\beta$ (see Fig. 2(a)). The single wavelength component will acquire a tilt angle due to the difference $(Q+R)$ in path lengths between the edges of the diffracted beam spot $s$. One can calculate the tilt angle $\gamma$ of this single wavelength component of the pulse front incident on the grating as

$$
\tan \left(\frac{\pi}{2}-\gamma\right)=\frac{s}{Q+R}=\frac{\cos \beta}{\sin \alpha+\sin \beta}
$$

The denominator of (5) corresponds to the grating equation:

$$
\sin \alpha+\sin \beta=m p \lambda_{0}
$$

where $m$ and $p$ are respectively the diffraction order and groove density of a grating, and $\lambda_{0}$ the central wavelength for the pump pulse. The tilt angle $\gamma_{1}$ of the pulse after a two-lens telescope with a magnification $M_{\mathrm{g}}=f_{2} / f_{1}$ can be also expressed by (see Fig. 2(b)):

$$
\tan \left(\frac{\pi}{2}-\gamma_{1}\right)=M_{g} \tan \left(\frac{\pi}{2}-\gamma\right) .
$$

The magnification $M_{\mathrm{g}}$ can be also defined by $M_{\mathrm{g}}=s_{1} / s$ and thus expressed by the following equation:

$$
M_{g}=\frac{s_{1}}{s}=\frac{t_{1}}{t \tan \gamma_{c} \tan \left(\frac{\pi}{2}-\gamma\right)} .
$$

By using (5)-(8) and considering the pulse front length decreases in LN crystal, i.e. $t_{1}=t / n_{N I R}^{g}$, the tilt angle $\gamma_{\mathrm{c}}$ in LN can be derived as follows:

$$
\tan \gamma_{c}=\frac{m \lambda_{0} p}{n_{N I R}^{g} M_{g} \cos \beta}
$$

The THz polariton generated by the tilted pulse front of the pump propagates perpendicularly to the front in the LN crystal (Fig. 1(b)), and it is coupled out into free space through the surface of the crystal cut at the tilt angle $\theta_{\mathrm{LN}}\left(=\gamma_{\mathrm{c}}\right)$ (Fig. 2(b)). 


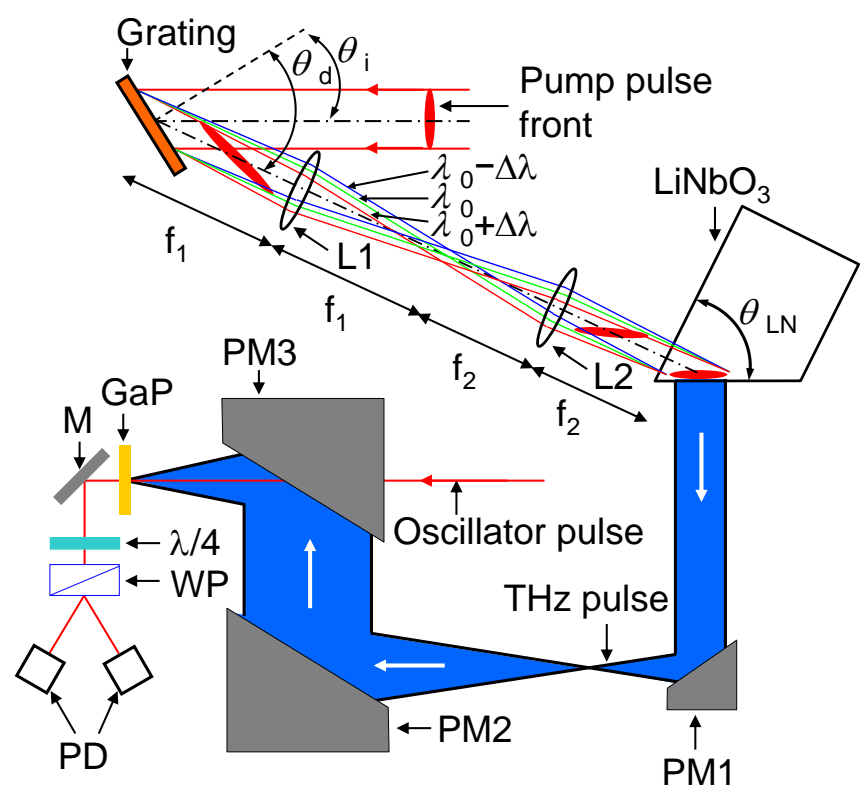

Fig. 3. THz pulse generation using the tilted-pump-pulse-front scheme and electro-optic sampling setup. The $4 f$-lens configuration consists of two cylindrical lenses L1 and L2 with focal lengths of $250 \mathrm{~mm}$ and 150 $\mathrm{mm}$ in the horizontal direction. The grating has 1800 lines $/ \mathrm{mm}$. The incident angle $\theta_{\mathrm{i}}$ and diffracted angle $\theta_{\mathrm{d}}$ of the grating are $35^{\circ}$ and $56^{\circ}$. The LN prism angle $\theta_{\mathrm{LN}}$ is $62^{\circ}$. The off-axis parabolic mirrors PM1, PM2, and PM3 have effective focal lengths of 10, 100, and $50 \mathrm{~mm}$ and diameters of 10, 50, and $50 \mathrm{~mm}$. M: mirror, WP: Wollaston prism, PD: photo detector

\section{3) $\mathrm{THz}$ generation and detection setup}

Fig. 3 shows the tilted-pulse-front pumping scheme for $\mathrm{THz}$ pulse generation and detection using electro-optic (EO) sampling [48]. The source is an amplified Ti:sapphire laser (pulse energy: $4 \mathrm{~mJ}$, FWHM: $85 \mathrm{fs}$, central wavelength: 780 $\mathrm{nm}$, repetition rate: $1 \mathrm{kHz}$ ). The EO sampling uses optical pulses from an $80-\mathrm{MHz}$ oscillator synchronized with the amplified pulses.

The intensity front of the pump pulse is tilted with a grating. A pair of cylindrical lenses in the $4 f$-lens geometry is used to tilt the pulse more (see (7)) and to image the pump spot onto the LN crystal (horizontal magnification $M_{\mathrm{g}}$ : 0.6) [69]. The angles of the incident and first-order diffracted beams are set to $\alpha=35.3^{\circ}$ and $\beta=55.7^{\circ}$ to get a tilt angle $\theta_{\mathrm{c}}$ of $62^{\circ}$ and ensure efficient generation around $1 \mathrm{THz}$. A $\lambda / 2$ plate changes pump beam from horizontal (for which the grating has high efficiency) to vertical polarization aligned with the optic axis of the LN crystal (not shown in Fig. 3); the $d_{33}$ electro-optic tensor component of $\mathrm{LN}$ is the largest. The beam is focused onto the crystal by using three off-axis parabolic mirrors PM1, PM2, and PM3.

Fig. 4(a) shows the temporal profile measured with a 300$\mu$ m-thick GaP detection crystal. Here, six high-resistivity $\mathrm{Si}$ attenuators were put in front of the crystal to reduce the field amplitude. The maximum modulation of balanced photodetector signals measured at the peak $\mathrm{THz}$ field was 0.44 ; this value corresponds to an electric field of $1.2 \mathrm{MV} / \mathrm{cm}$ without $\mathrm{Si}$ attenuators. The inset shows a $\mathrm{THz}$ image at the

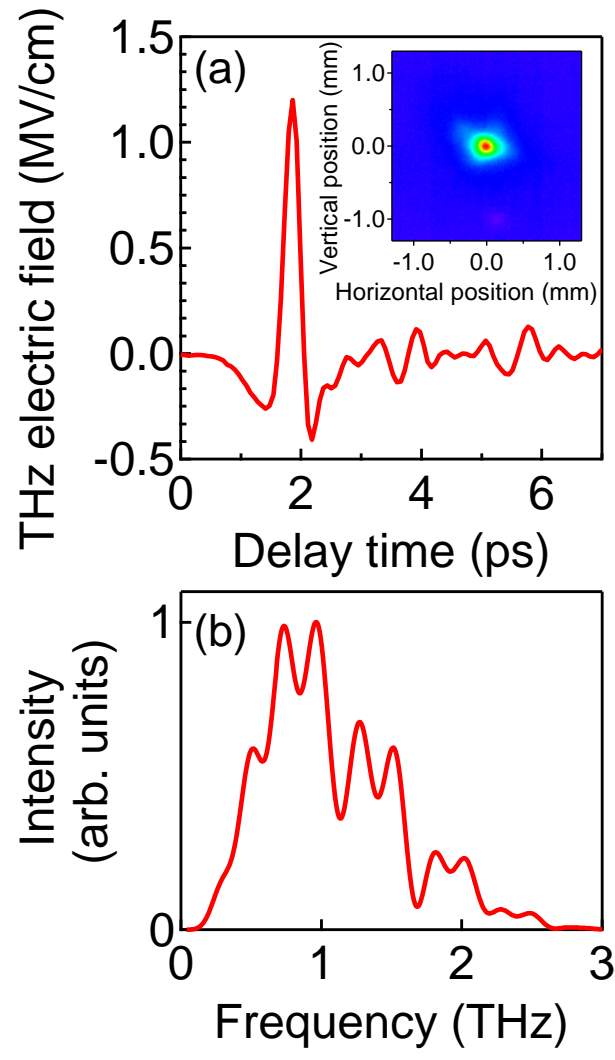

Fig. 4. (a) Measured $\mathrm{THz}$ temporal profile and (b) its Fourier spectrum. Inset in (a): THz image measured at the focus of PM3.

focus after PM3. The spectrum (Fig. 4(b)) has a maximum around $0.8 \mathrm{THz}$ and has water-vapor absorption lines (no dry air purging).

An uncooled microbolometer $\mathrm{THz}$ camera (NEC Corporation, IRV-T0830) picked up the intensity image from pulses passing through a 300-GHz-width band-pass filter for 1 THz (Murata Manufacturing Co., Ltd., MMBPF40-1000). The small spot (FWHM intensity: $300 \mu \mathrm{m}$ ) is near the diffraction limit. The total pulse energy was estimated to be $\sim 2 \mu \mathrm{J}$ by integrating the $\mathrm{THz}$ intensity temporally and spatially [75], and the energy conversion efficiency was $\sim 10^{-3}$.

\section{NONPERTURBATIVE EXCITONIC INTERACTION WITH INTENSE THZ PULSES}

The nonlinear interaction of matter with strong, oscillating electric fields has attracted considerable attention because of their importance in fundamental physics and technological applications. The resonant (or nearly resonant) interaction of electric fields with electronic transitions in semiconductors and atomic gases exhibits intriguing nonlinear phenomena such as the ac (optical) Stark effect [76,77], Rabi oscillations [78], Autler-Townes splitting [40,42,76], and electromagnetically induced transparency [79]. Even in nonresonant situations, atomic systems subjected to highintensity laser excitation exhibit extreme nonlinear phenomena such as above-threshold ionization [80] and high-order 


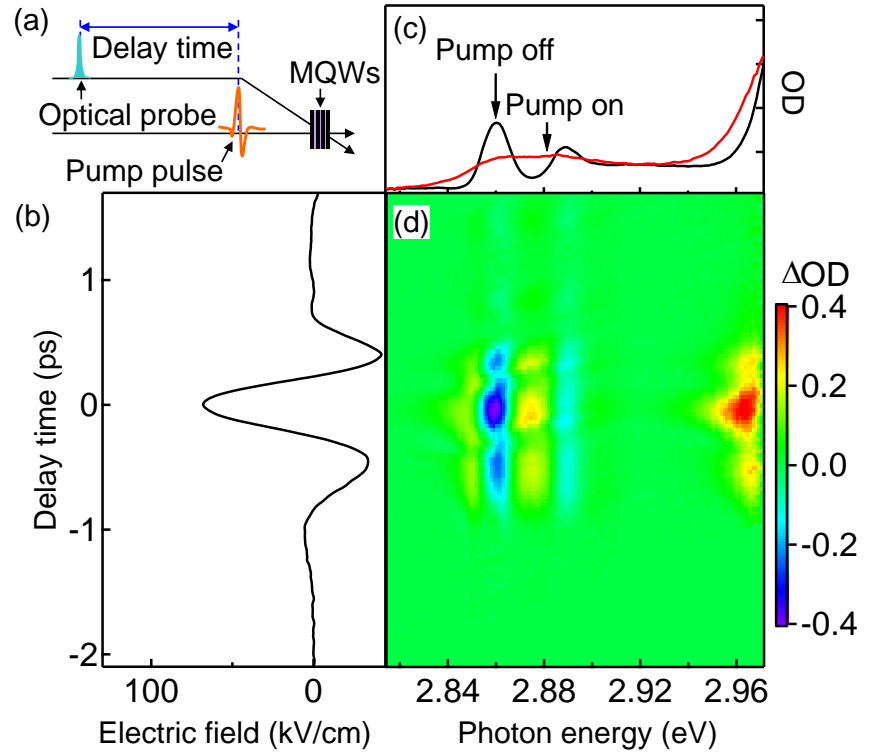

Fig. 5. (a) THz-pump and optical-probe experimental setup. The linear polarization directions of the pump and probe pulses are parallel to the MQW layers. (b) Observed $\mathrm{THz}$ temporal profile. (c) Excitonic absorption spectrum observed at pump-probe delays of -1.8 (THz pump off) and $0 \mathrm{ps}$ ( $\mathrm{THz}$ pump on). (d) Differential absorption spectrum plotted a function of optical energy and delay between THz-pump and optical-probe pulses. Figure adapted from Ref. [60].

harmonic generation [81-85], and these phenomena cannot be described by perturbation theory.

The key concept behind these nonperturbative phenomena is ponderomotive energy, which is the cycle-averaged kinetic energy of an electron wiggling in an oscillating electric field [86], i.e.,

$$
U_{P}=\frac{e^{2} E^{2}}{4 m^{*} \omega^{2}}
$$

where $E$ is the electric-field amplitude of the incident laser pulse, $e$ is the elementary charge, $m^{*}$ is effective mass, and $\omega / 2 \pi$ is the laser field's frequency. Since ponderomotive energy increases quadratically with the inverse square of frequency, even small photon energies can enhance nonperturbative effects. A terahertz $(\mathrm{THz})$ electric field could make the poderomotive energy larger than that of a visible light source. A lower effective mass in semiconductors could also enhance it [87].

Fig. 5(a) illustrates the THz-pump and optical-probe experiment using the tilted pump pulse front technique. The multiple quantum wells (MQW) sample was grown on a (100) GaAs substrate; the MQW consisted of 30 repetitions of 5-nmZnSe wells and 30-nm-ZnMgSSe barriers. Fig. 5(b) shows the $\mathrm{THz}$ temporal profile measured via EO sampling. The THzpulse beam at the focus was set to $1.5 \mathrm{~mm}$ in diameter using a $90^{\circ}$ off-axis parabolic mirror (focal length: $100 \mathrm{~mm}$ ). The maximum field at this position was about $68 \mathrm{kV} / \mathrm{cm}$. For the probe pulse, we used a white-light continuum generated by

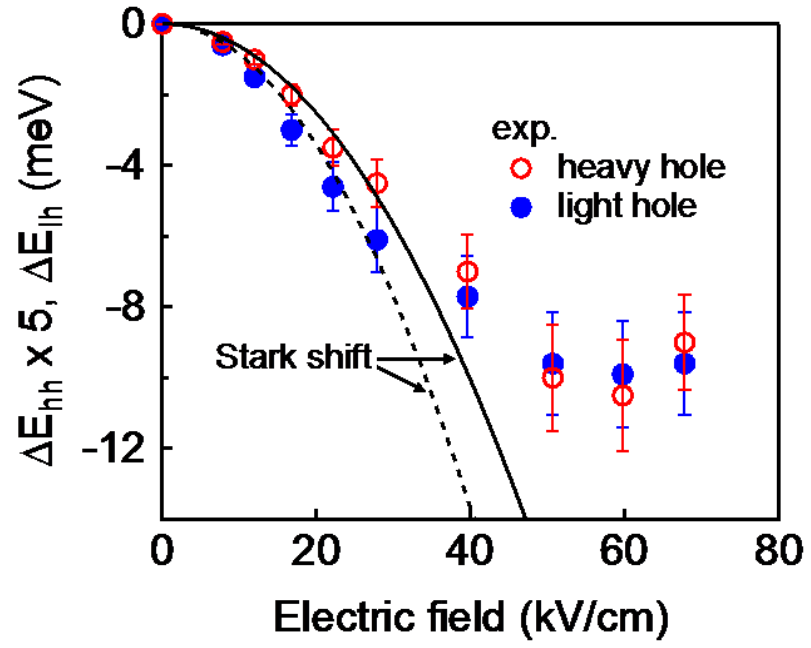

Fig. 6. Absorption peak energy of $h h$ (open circles) and $l h$ (solid circles) excitons versus the incident $\mathrm{THz}$ field $E_{\mathrm{THz}}$. Solid and dashed lines are fits of the Stark effect to the data for the $h h$ and $l h$ excitons, respectively. Figure adapted from Ref. [60].

focusing a small portion of the laser pulses into a 10-mm-thick quartz cell containing water. The resulting continuum spectrum ranged from the visible to near-infrared.

Fig. 5(d) presents the differential absorption spectrum as a function of optical energy and delay between the THz-pump and optical-probe pulses. Fig. 5(c) shows the optical absorption spectrum with the $\mathrm{THz}$ pump off and on (i.e., at pump-probe delays of -1.8 and $0 \mathrm{ps}$ ). The absorption peaks at 2.86 and $2.89 \mathrm{eV}$ in the pump-off spectra in Fig. 5(c) are respectively due to $n=1$ heavy-hole $(h h)$ and light-hole $(l h)$ excitonic absorptions. These excitonic resonances are strongly modulated and suppressed by the THz pump. The absorption increases below the band edge of the $\mathrm{ZnMgSSe}$ (bulk barrier layer) and above $2.92 \mathrm{eV}$. This behavior is attributed to the dynamical Franz-Keldysh effect (DFKE) [88-90].

The suppression of excitonic absorptions in Fig. 5(c) indicates that the $\mathrm{THz}$ electric field distorts the Coulomb potential between the electron and hole of the exciton and a widens the Coulomb well, which induces the $1 s$ level to shift to lower energies. Fig. 6 shows the peak energy shift of the $h h$ - and $l h$-excitonic absorptions. The peaks redshift monotonously until $\sim 40 \mathrm{kV} / \mathrm{cm}$, at which point the redshift changes to a blueshift.

The perturbative Stark-effect theory is applicable in the weak-field limit up to $\sim 40 \mathrm{kV} / \mathrm{cm}$. The departure of the experimental data from the quadratic Stark shift at stronger fields and the appearance of a blueshift implies competition between two different nonperturbative phenomena: 1) the high $\mathrm{THz}$ field exceeds the electric field necessary to ionize the $h h$ exciton (estimated from $E_{\mathrm{B}} / e a_{\mathrm{B}}$ to be $\sim 44 \mathrm{kV} / \mathrm{cm}$ ), and thus, the $\mathrm{THz}$ field dominates the excitonic Coulomb potential and induces the mixing of excitonic states with continuum states; 2) The DFKE causes a blueshift of the band-edge of the $2 \mathrm{D}$ electronic continuum states [88]. 


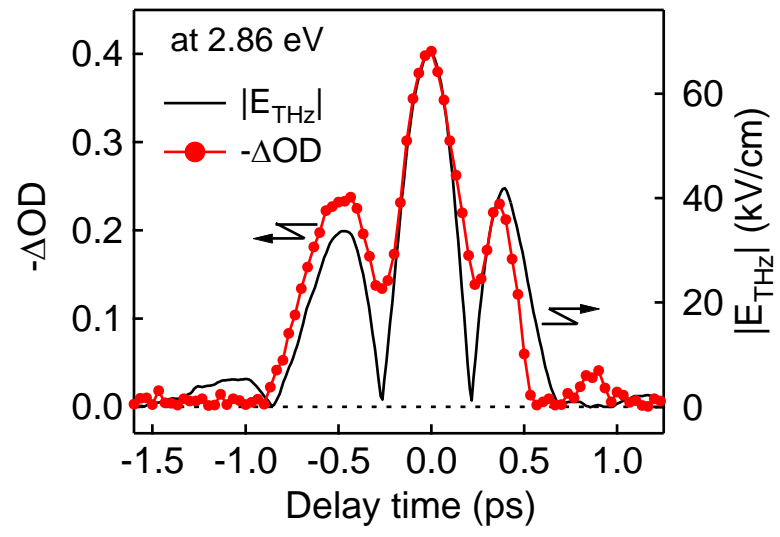

Fig. 7. Temporal profiles of the absorption change in the vicinity of the hh exciton (at $2.86 \mathrm{eV}$ ) and the absolute value of the electric field of the $\mathrm{THz}$ pulse. Figure adapted from Ref. [60].

The optical temporal response of excitons in quantum wells to the THz pulses may be able to be exploited for ultrafast optical modulation. Fig. 7 compares the temporal profiles of the absorption change in the vicinity of the $h h$ exciton (at 2.86 $\mathrm{eV}$ ) and the absolute value of the pulse's electric field. The change follows the temporal profile rather closely. Theory based on a one-dimensional exciton predicts that the effective response time is just the exciton dephasing time and is the reciprocal of homogeneous broadening [91]. The dephasing time at zero field has been measured by femtosecond fourwave mixing to be $\sim 450 \mathrm{fs}$ [92], and it would be shortened by applying a $\mathrm{THz}$ field. Thus, the subpicosecond electroabsorption response time for excitons implied by the data of Fig. 7 is reasonable. The efficient modulation of the excitonic absorption and the short response time are promising for Tbit/s wireless transmission systems.

\section{CARrier Generation by INTENSE Terahertz PUlse EXCITATION}

Cutoff frequencies of electronic devices are getting close to $1 \mathrm{THz}$, and carriers may experience electric fields up to 1 $\mathrm{MV} / \mathrm{cm}$. In fact, metal electrodes with a $10 \mathrm{~nm}$ gap would have such a high field between them if a bias voltage of $1 \mathrm{~V}$ were applied. This means the high-field nonlinear transport properties of semiconductors can no longer be ignored in engineering [93-96]. Although the high-field properties that affect transport on the nanometer and ultrashort time-period scales are not well understood, they can be investigated with intense $\mathrm{THz}$ pulse sources [97-103].

Carrier multiplication via impact ionization under high electric fields underlies many nonlinear transport phenomena. It plays a key role in conduction breakdown in transistors $[93,104,105]$, the avalanche effect in single-photon detectors [106], and in the workings of electroluminescent emitters [107-109] and solar cells [110-112]. In the process [94] illustrated Fig. 8(a), a highly energetic conduction-band electron $\left(e_{11}\right)$ colliding with a valence-band electron yields two conduction electrons $\left(e_{12}+e_{22}\right)$ and a hole $\left(h_{21}\right)$. The electron $e_{11}$ gains energy in the strong electric field, and this energy (a)

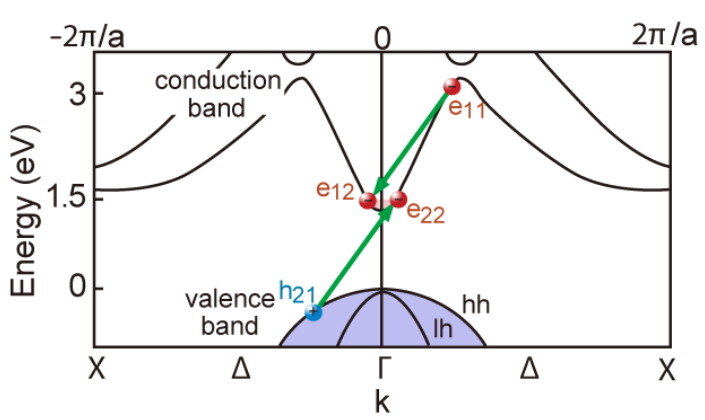

(b)

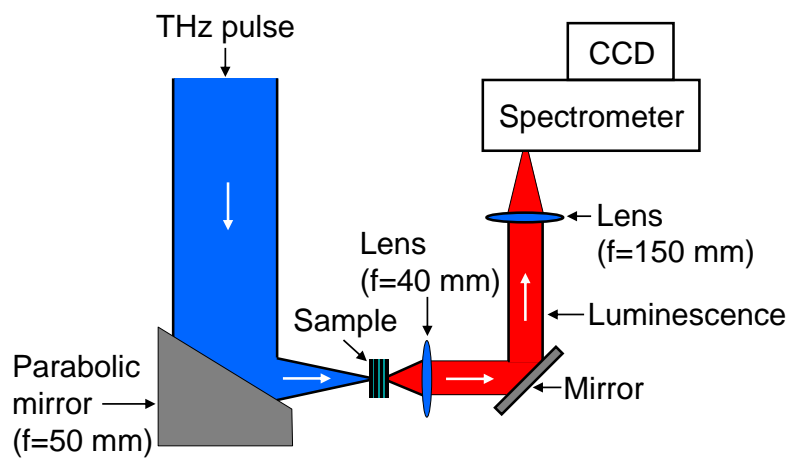

Fig. 8. (a) Electron-initiated impact ionization transitions in GaAs band structure in the $\Delta$ direction. The lattice constant $a$ of GaAs is $5.6 \AA$, and $\pm 2 \pi / a$ corresponds to $\pm 1.1 \times 10^{10} \mathrm{~m}^{-1}$. The diagram shows electrons and hole positions before and after the transition at the threshold. (b) Generated $\mathrm{THz}$ pulses are focused onto the GaAs QWs sample, and the luminescence is detected by a CCD camera after it has passed through the spectrometer. Figure adapted from Ref. [61].

gain implies that carriers driven without being affected by incoherent phonon scattering acquire enough kinetic energy to trigger a series of impact ionizations, i.e., carrier multiplication.

Meanwhile, electrons in the conduction band of GaAs move ballistically without incoherent LO-phonon scattering for hundreds of femtoseconds ( $115 \mathrm{fs}, \omega_{\mathrm{LO}}=$ angular frequency of the LO phonon) [97]. Carriers accelerated ballistically on ultra-short timescales can therefore be expected to gain kinetic energy more efficiently and thereby trigger carrier multiplication. However, insufficiently strong $\mathrm{THz}$ pulses generate few carriers [101], and various additional phenomena induced by them, such as phonon absorption [102], intervalley scattering [98], and exciton dissociation [60,113], obscure the carrier multiplication.

We developed an experimental scheme for studying carrier multiplication in nominally undoped GaAs MQW (Fig. 8(b)). Carrier multiplication was induced by irradiating the QWs with a nearly half-cycle $\mathrm{THz}$ pulse (1 ps, max. electric field: $1.05 \mathrm{MV} / \mathrm{cm}$ ) [48] (see Fig. 4). The electric field was perpendicular to the stacking direction (100) of the sample. The field ionization due to the $\mathrm{THz}$ pulses accelerates electrons from residual impurity donors along the $\Delta$ line around the Brillouin zone center $(\Gamma$ point $)$ in momentum space (Fig. 8(b)) [114]. When the electric field reaches a certain strength, the electrons acquire enough energy to induce several impact ionizations and produce many electron-hole $(e-h)$ pairs that go on to form excitons around the $\Gamma$ point. The impulsive 
(a)

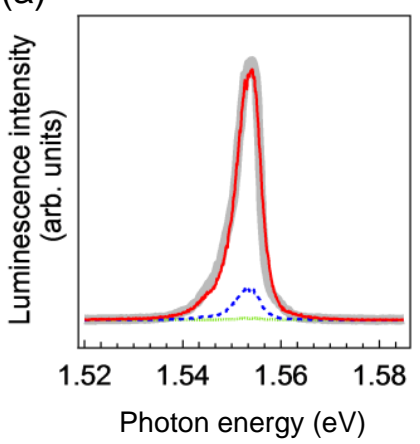

(b)

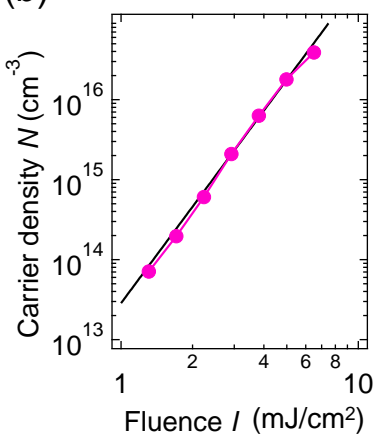

Fig. 9. (a) Spectra of luminescence excited at $10 \mathrm{~K}$ by THz pulses with peak amplitudes of $0.54 \mathrm{MV} / \mathrm{cm}$ (green dotted line), $0.70 \mathrm{MV} / \mathrm{cm}$ (blue dashed line), $1.05 \mathrm{MV} / \mathrm{cm}$ (red solid line), and by optical pulse (3.18 eV, gray solid line). (b) $\mathrm{THz}$ pulse excitation intensity dependence of carrier density (magenta closed circles). The eye guide (black solid line) corresponds to a fourth-power-law $\mathrm{THz}$ intensity dependence. Figure adapted from Ref. [61].

electric field allows unbound $e-h$ pairs to form excitons that decay radiatively because the electric field that would otherwise ionize the exciton is absent just after the pairs are generated [115]. Luminescence from the excitons is detected by a liquid-nitrogen-cooled CCD camera (Fig. 8(b)).

Fig. 9(a) shows that $\mathrm{THz}$ pulses with different field strengths induce near-infrared luminescence centered around $1.55 \mathrm{eV}$ from MQW at $10 \mathrm{~K}$, even though the central photon energy of the pulse $(\sim 4 \mathrm{meV})$ is about 390 times lower than the luminescence photon energy (see Fig. 4), and the luminescence intensity drastically decreases as the electric field $\varepsilon$ decreases. Since luminescence is proportional to the created $e$ - $h$ pair density [116], the generated carrier density $N$ can be estimated by comparing the luminescence measurement with optical pulse excitation measurements. The carrier density $N$ plotted as a function of $\mathrm{THz}$ pulse fluence $I$ is extremely nonlinear and roughly proportional to $I^{4}\left(\propto \varepsilon^{8}\right)$ (Fig. 9(b)). The minimum and maximum fluences correspond to 0.47 and $1.05 \mathrm{MV} / \mathrm{cm}$, and the carrier density increases over this range by about three orders of magnitude. As shown in Fig. 9(a), the spectral shapes are almost the same, and the peak photon energies are near the $h h$ exciton absorption peak.

We can attribute the substantial numbers of carriers to carrier multiplication triggered by impact ionizations rather than to Zener tunneling because the tunneling occurring under a $1 \mathrm{MV} / \mathrm{cm}$ DC electric field lasting $1 \mathrm{ps}$ can account for only a small amount of the $e-h$ pairs $\left(10^{11} \mathrm{~cm}^{-3}\right)$ [117]. In the impact ionization process, an electron in the conduction band $\left(e_{11}\right)$ gain kinetic energy from the electric field and creates an electron $\left(e_{12}\right)$ plus an $e$ - $h$ pair $\left(e_{22}+h_{21}\right)$ (Fig. 8(a)):

$$
e_{11} \rightarrow e_{12}+e_{22}+h_{21} \text {. }
$$

This process is possible only if the electron has a threshold kinetic energy higher than the band gap $E_{\mathrm{g}}$ of $1.52 \mathrm{eV}$. As shown in Fig. 8(a), if an electron released from an impurity donor is accelerated to the threshold kinetic energy, it can create an $e-h$ pair. Subsequently, both the electron that lost energy to create the $e-h$ pair and the created $e-h$ pair could, in (a)

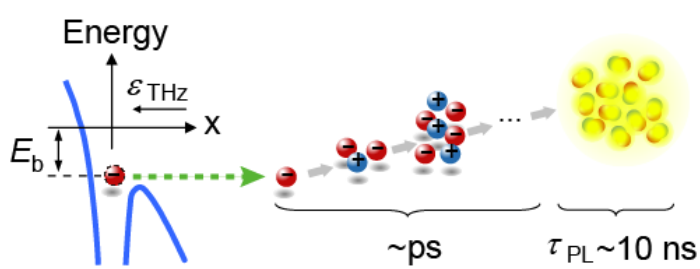

(b)

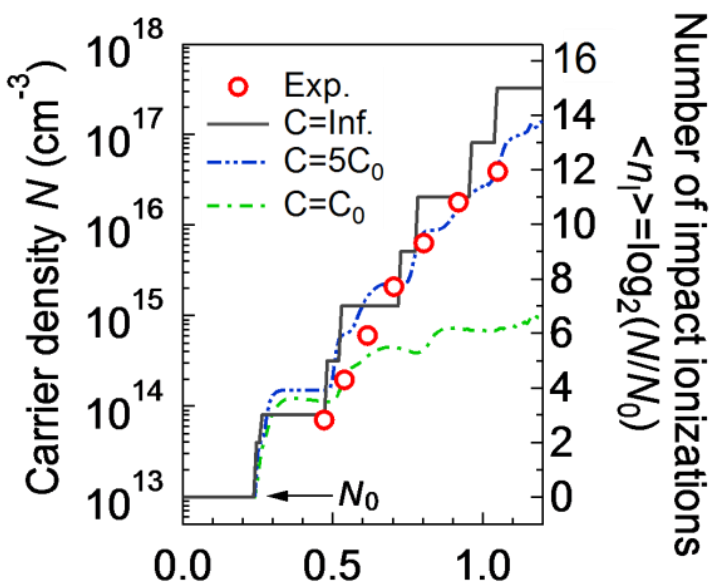

\section{THz electric field $(\mathrm{MV} / \mathrm{cm})$}

Fig. 10. (a) Sketch visualizing the distortion in the Coulomb potential of donors, causing the potential to widen and free electrons to be released, and subsequent evolution of unbound $e-h$ gas generated by a series of impact ionizations into a pure population of excitons emitting luminescence. (b) Electric field dependences of carrier density obtained in the experiment (red open circles) and calculations using equation (12) for three different impact ionization rates $\gamma_{I}^{c a l}$; one is infinity (gray solid line), and the others are derived from (13) with $C=C_{0}=870 \mathrm{ps}^{-1} \mathrm{eV}^{-2}$ (green one-dot-dashed line) and $C=5 C_{0}$ (blue two-dot-dashed line). The initial carrier density was assumed to be $N_{0}=10^{13} \mathrm{~cm}^{-3}$. For the experimental (red open circles) and calculated data (gray solid line), the carrier density $N$ is plotted together with the corresponding $\left\langle n_{\mathrm{I}}\right\rangle$. Figure adapted from Ref. [61].

turn, gain energy from the field. This process can continue as long as the electric field of the THz pulse remains sufficiently strong, and so, comparably few impact ionization events can nonetheless produce numerous $e$ - $h$ pairs (Fig. 10(a)).

These multiplication dynamics can be compared with experimental observation. As can be seen from (11), an electron-initiated impact ionization event doubles the number of electrons. That is, given an initial electron density $N_{0}$, the electron and hole densities after $\left\langle n_{\mathrm{I}}\right\rangle$ impact ionization events are respectively $N_{0} \times 2^{\left\langle n_{I}\right\rangle}$ and $N_{0} \times\left(2^{\left\langle n_{I}\right\rangle}-1\right)$. Thus, the increase in impact ionizations $\left\langle\Delta n_{\mathrm{I}}\right\rangle$ due to the electric field changing from $\varepsilon_{\min }=0.47$ to $\varepsilon_{\max }=1.05 \mathrm{MV} / \mathrm{cm}$ is $\log _{2}\left(N\left(\varepsilon_{\max }\right) / N\left(\varepsilon_{\min }\right)\right) \sim 9$ (Fig. 10(b)). That is, at least nine impact ionizations on average occur within the 0.5 -ps duration of the positive lobe of the $\mathrm{THz}$ pulse. Hence, the impact ionization rate $\gamma_{\mathrm{I}}$ at the maximum electric field is approximately $9 / 0.5 \times 10^{-12} \mathrm{~s} \sim 2 \times 10^{13} \mathrm{~s}^{-1}$.

The experimentally obtained impact ionization rate $\gamma_{\text {I }}$ can be quantitatively compared with a simple theoretical 
prediction whereby the electron motion in an electric field follows the dispersion of the band structure in momentum space:

$$
\hbar \frac{d k(t)}{d t}=-e \varepsilon(t)
$$

where $e$ is the electron charge and $\hbar$ is Planck's constant. Here, the electric field is of a pulse reflected a number of times inside the sample. The change in wavenumber $k(t)$ during the pulse is calculated assuming that the electrons lose all their kinetic energy when they reach the average wavenumber $\pm 2.77 \times 10^{9} \mathrm{~m}^{-1}$, that is, an infinite impact ionization rate. The theoretical increase in number $\left\langle\Delta n_{\mathrm{I}}\right\rangle$ from $\varepsilon_{\min }=0.47$ to $\varepsilon_{\max }=$ $1.05 \mathrm{MV} / \mathrm{cm}$ is $\left\langle n_{\mathrm{I}}\left(\varepsilon_{\max }\right)\right\rangle-\left\langle n_{\mathrm{I}}\left(\varepsilon_{\min }\right)\right\rangle=12$, which is close to the experimentally obtained $\left\langle\Delta n_{\mathrm{I}}\right\rangle$, and this confirms the experimentally derived $\gamma_{\mathrm{I}}$ of $\sim 10^{13} \mathrm{~s}^{-1}$.

We can also analyze the carrier density with a finite impact ionization rate using the Keldysh formula [118]:

$$
\gamma_{I}^{c a l}=C\left(E-E_{t h}\right)^{2}, \quad E>E_{t h} .
$$

The corresponding values for electrons are taken from Ref. [118]: $C=C_{0}=870 \mathrm{ps}^{-1} \mathrm{eV}^{-2}$ and $E_{\mathrm{th}}=1.7 \mathrm{eV}$. As shown in Fig. 10(b), putting $C=5 C_{0}$ in (13) (blue two-dot-dashed line) reproduces the experimentally observed carrier density and is close to the curve for the infinite rate (gray solid line). Here, $\gamma_{I}^{\text {cal }}$ is $2.5 \times 10^{14} \mathrm{~s}^{-1}$ (derived by using (13) with $C=5 C_{0}$ and $E=1.94 \mathrm{eV}$ at the maximum first-conduction-band energy between the $\Gamma$ and $X$ points; see Fig. 8(a)). The experimentally obtained value $\left(\gamma_{\mathrm{I}} \sim 10^{13} \mathrm{~s}^{-1}\right)$ is almost ten times smaller because electrons took time to accelerate to $E_{\mathrm{th}}$.

We demonstrated extraordinarily high carrier multiplication in GaAs subjected to a $\mathrm{THz}$ pulse of a strong electric field; the carriers increased by about three orders of magnitude. The increase can be explained with a phenomenological impact ionization model including electron motion in $k$-space with a pristine band structure. A full quantum kinetic treatment of carrier dynamics in a band structure modified by an intense $\mathrm{THz}$ electric field should be performed to reveal the microscopic origin of the carrier multiplication [119-121]. Our findings of efficient ultrafast carrier multiplication bode well for future ultrahigh-speed device applications such as highquantum-efficiency THz-biased avalanche photodiodes that have femtosecond resolution and are sensitive to a single photon. They may also lead to more efficient electroluminescent and photovoltaic nanoscale devices.

\section{CONCLUSION}

The tilted-pump-pulse-front scheme using a $\mathrm{LiNbO}_{3}$ crystal can generate intense single-cycle $\mathrm{THz}$ pulses whose nonlinear interaction with semiconductors may have many novel applications. In particular, intense single-cycle $\mathrm{THz}$ pulses induce strong spectral modulations in the excitonic and bandedge absorption of $\mathrm{ZnSe} / \mathrm{ZnMgSSe} \mathrm{MQW}$. Furthermore, the extremely high $(1 \mathrm{MV} / \mathrm{cm})$ electric field of a $\mathrm{THz}$ pulse allows us to excite electrons from the valence to conduction band of GaAs/AlGaAs MQW and observe exciton luminescence.

\section{ACKNOWLEDGMENT}

The authors would like to thank many colleagues and graduate students for their contributions and discussions. In particular K. Shinokita, A. Doi, F. Blanchard, M. Shirai, S. Tani, M. Nagai, Y. Kadoya, and T. Aoki deserve special mention here. This work was supported by KAKENHI (Grant Nos. 21760038 and 20104007) and Grant-in-Aid for Creative Scientific Research (Grant No. 18GS0208) of the Ministry of Education, Culture, Sports, Science and Technology, Japan.

\section{REFERENCES}

[1] M. van Exter, C. Fattinger, and D. Grischkowsky, "Terahertz timedomain spectroscopy of water vapor," Opt. Lett., vol. 14, pp. 1128-1130, 1989

[2] D. Grischkowsky, S. Keiding, M. van Exter, and C. Fattinger, "Farinfrared time-domain spectroscopy with terahertz beams of dielectrics and semiconductors," J. Opt. Soc. Am. B, vol. 7, pp. 2006-2015, 1990.

[3] D. Mittleman, Sensing with Terahertz Radiation. Berlin, Germany: Springer-Verlag, 2003

[4] K. Sakai, Terahertz Optoelectronics. Berlin, Germany: Springer-Verlag, 2005.

[5] P. U. Jepsen, D. G. Cooke, and M. Koch, "Terahertz spectroscopy and imaging-Modern techniques and applications," Laser Photonics Rev., vol. 5, pp. 124-166, 2011.

[6] P. R. Smith, D. H. Auston, and M. C. Nuss, "Subpicosecond photoconducting dipole antennas," IEEE J. Quantum. Electron. vol. 24, pp. 255-260, 1998.

[7] C. Fattinger and D. Grischkowsky, "THz beams," Appl. Phys. Lett., vol. 54, pp. 490-492, 1989.

[8] D. H. Auston and P. R. Smith, "Generation and detection of millimeter waves by picosecond photoconductivity," Appl. Phys. Lett., vol. 43, pp. 631-633, 1983.

[9] S. Kono, M. Tani, and K. Sakai, "Ultrabroadband photoconductive detection: Comparison with free-space electro-optic sampling," Appl. Phys. Lett. vol. 79, pp. 898-900, 2001.

[10] D. H. Auston, K. P. Cheung, J. A. Valdmanis, and D. A. Kleinman, "Cherenkov radiation from femtosecond optical pulses in electro-optic media," Phys. Rev. Lett. vol. 53, pp. 1555-1562, 1984.

[11] Q. Wu and X.-C. Zhang, "Free-space electro-optic sampling of terahertz beams," Appl. Phys. Lett., vol. 67, pp. 3523-3525, 1995.

[12] Q. Wu and X.-C. Zhang, "Ultrafast electro-optic field sensors," Appl. Phys. Lett., vol. 68, pp. 1604-1606, 1996.

[13] P. U. Jepsen, C. Winnewisser, M. Schall, V. Schyja, S. R. Keiding, and H. Helm, "Detection of $\mathrm{THz}$ pulses by phase retardation in lithium tantalate," Phys. Rev. E, vol. 53, pp. 3052-3054(R), 1996.

[14] A. Nahata, D. H. Auston, T. F. Heinz, and C. J. Wu, "Coherent detection of freely propagating terahertz radiation by electro-optic sampling," Appl. Phys. Lett., vol. 68, pp. 150-152, 1996.

[15] C. Fattinger and D. Grischkowsky, "Point source terahertz optics," Appl. Phys. Lett., vol. 53, pp. 1480-1482, 1998.

[16] J. T. Darrow, B. B. Hu, X.-C. Zhang, and D. H. Auston, "Subpicosecond electromagnetic pulses from large-aperture photoconducting antennas," Opt. Lett., vol. 15, pp. 323-325, 1990.

[17] Q. Wu and X.-C. Zhang, "7 terahertz broadband GaP electro-optic sensor," Appl. Phys. Lett., vol. 70, pp. 1784-1786, 1997.

[18] Q. Wu and X.-C. Zhang, "Free-space electro-optics sampling of midinfrared pulses," Appl. Phys. Lett., vol. 71, pp. 1285-1286, 1997.

[19] R. Huber, A. Brodschelm, F. Tauser, and A. Leitenstorfer, "Generation and field-resolved detection of femtosecond electromagnetic pulses tunable up to 41 THz," Appl. Phys. Lett., vol. 76, pp. 3191-3193, 1997.

[20] R. A. Kaindl, F. Eickemeyer, M. Woerner, and T. Elsaesser, "Broadband phase-matched difference frequency mixing of femtosecondpulses in GaSe: Experiment and theory," Appl. Phys. Lett., vol. 93, pp. 1060-1062, 1999. 
[21] A. Sell, R. Scheu, A. Leitenstorfer, and R. Huber, "Field-resolved detection of phase-locked infrared transients from a compact Er:fiber system tunable between 55 and 107 THz," Appl. Phys. Lett., vol. 93, pp. 251107-1-251107-3, 2008.

[22] R. Huber, F. Tauser, A. Brodschelm, M. Bichler, G. Abstreiter, and A. Leitenstorfer, "How many-particle interactions develop after ultrafast excitation of an electron-hole plasma," Nature, vol. 414, pp. 286-289, 2001.

[23] R. A. Kaindl, M. A. Carnahan, D. Hägele, R. Lövenich, and D. S. Chemla, "Ultrafast terahertz probes of transient conducting and insulating phases in an electron-hole gas," Nature, vol. 423, pp. 734-738, 2003.

[24] T. Kampfrath, L. Perfetti, F. Schapper, C. Frischkorn, and M. Wolf, "Strongly coupled optical phonons in the ultrafast dynamics of the electronic energy and current relaxation in graphite," Phys. Rev. Lett., vol. 95, pp. 187403-1-187403-4, 2005.

[25] M. Kubouchi, K. Yoshioka, R. Shimano, A. Mysyrowicz, and M. Kuwata-Gonokami, "Study of orthoexciton-to-paraexciton conversion in $\mathrm{Cu}_{2} \mathrm{O}$ by excitonic Lyman spectroscopy," Phys. Rev. Lett., vol. 94, pp. 016403-1-016403-4, 2005.

[26] C. Kübler, H. Ehrke, R. Huber, R. Lopez, A. Halabica, R. F. Haglund, Jr., and A. Leitenstorfer, "Coherent structural dynamics and electronic correlations during an ultrafast insulator-to-metal phase transition in $\mathrm{VO}_{2}$," Phys. Rev. Lett., vol. 99, pp. 116401-1-116401-4, 2007.

[27] E. Hendry, F. J. Garcia-Vidal, L. Martin-Moreno, J. G. Rivas, M. Bonn, A. P. Hibbins, and M. J. Lockyear, "Optical control over surfaceplasmon-polariton-assisted $\mathrm{THz}$ transmission through a slit aperture," Phys. Rev. Lett., vol. 100, pp. 123901-1-123901-4, 2008.

[28] T. Suzuki and R. Shimano, "Time-resolved formation of excitons and electron-hole droplets in Si studied using terahertz spectroscopy," Phys. Rev. Lett. vol. 103, pp. 057401-1-1057401-4, 2009.

[29] A. Pashkin, M. Porer, M. Beyer, K. W. Kim, A. Dubroka, C. Bernhard, X. Yao, Y. Dagan, R. Hackl, A. Erb, J. Demsar, R. Huber, and A. Leitenstorfer, "Femtosecond response of quasiparticles and phonons in superconducting $\mathrm{YBa}_{2} \mathrm{Cu}_{3} \mathrm{O}_{7-\delta}$ studied by wideband terahertz spectroscopy," Phys. Rev. Lett., vol. 105, pp. 067001-1-067001-4, 2010.

[30] R. Ulbricht, E. Hendry, J. Shan, T. F. Heinz, M. Bonn, "Carrier dynamics in semiconductors studied with time-resolved terahertz spectroscopy," Rev. Mod. Phys., vol. 83, pp. 543-586, 2011.

[31] D. You, R. R. Jones, P. H. Bucksbaum, and D. R. Dykaar, "Generation of high-power sub-single-cycle 500-fs electromagnetic pulses," Opt. Lett., vol. 18, pp. 290-292, 1993.

[32] F. Blanchard, L. Razzari, H.C. Bandulet, G. Sharma, R. Morandotti, J. C. Kieffer, T. Ozaki, M. Reid, H. F. Tiedje, H. K. Haugen, and F. A. Hegmann, "Generation of $1.5 \mu \mathrm{J}$ single-cycle terahertz pulses by optical rectification from a large aperture ZnTe crystal," Opt. Express, vol. 15, pp. 13212- 13220, 2007.

[33] K. L. Yeh, M. C. Hoffmann, J. Hebling, and K. A. Nelson, "Generation of $10 \mu \mathrm{J}$ ultrashort terahertz pulses by optical rectification," Appl. Phys. Lett., vol. 90, pp. 171121-1171121-4, 2007.

[34] A. G. Stepanov, L. Bonacina, S. V. Chekalin, and J.-P. Wolf, "Generation of $30 \mu \mathrm{J}$ single-cycle terahertz pulses at $100 \mathrm{~Hz}$ repetition rate by optical rectification," Opt. Lett., vol. 33, pp. 2497- 2499, 2008.

[35] B. Ferguson and X.-C. Zhang, "Materials for terahertz science and technology," Nature Mater., vol. 1, pp. 26-33, 2002

[36] M. Tonouchi, "Cutting-edge terahertz technology," Nature Photon., vol. 1, pp. 97-105, 2007.

[37] F. Blanchard, A. Doi, T. Tanaka, H. Hirori, H. Tanaka, Y. Kadoya, and K. Tanaka, "Real-time terahertz near-field microscope," Opt. Express, vol. 19, pp. 8277-8284, 2011.

[38] A. Mayer and F. Keilmann, "Far-infrared nonlinear optics. I. $\chi^{(2)}$ near ionic resonance," Phys. Rev. B, vol. 33, 6954-6961, 1986.

[39] T. Dekorsy, V. A. Yakovlev, W. Seidel, M. Helm, and F. Keilmann, "Infrared-phonon-polariton resonance of the nonlinear susceptibility in GaAs," Phys. Rev. Lett., vol. 90, pp. 055508-1-055508-4, 2003.

[40] S. G. Carter, V. Birkedal, C. S. Wang, L. A. Coldren, A. V. Maslov, D. S. Citrin, M. S. Sherwin, "Quantum coherence in an optical modulator," Science, vol. 310, pp. 651-653, 2005.

[41] B. E. Cole, J. B. Williams, B. T. King, M. S. Sherwin, and C. R. Stanley, "Coherent manipulation of semiconductor quantum bits with terahertz radiation," Nature, vol. 410, pp. 60-63, 2001.

[42] M. Wagner, H. Schneider, D. Stehr, S. Winnerl, A. M. Andrews, S. Schartner, G. Strasser, and M. Helm, "Observation of the intraexciton Autler-Townes effect in GaAs/AlGaAs semiconductor quantum wells," Phys. Rev. Lett., vol. 105, pp. 167401-1- 167401-4, 2010.
[43] S. Ganichev and W. Prettl, Intense Terahertz Excitation of Semiconductors. Oxford: Oxford University Press, 2005.

[44] K. Reimann, R. P. Smi, A. M. Weiner, T. Elsaesser, and M. Woerner, "Direct field-resolved detection of terahertz transients with amplitudes of megavolts per centimeter," Opt. Lett., vol. 28, pp. 471-473, 2003.

[45] A. Sell, A. Leitenstorfer, and R. Huber, "Phase-locked generation and field-resolved detection of widely tunable terahertz pulses with amplitudes exceeding $100 \mathrm{MV} / \mathrm{cm}$," Opt. Lett., vol. 33, pp. 2767-2769, 2008

[46] F. Junginger, A. Sell, O. Schubert, B. Mayer, D. Brida, M. Marangoni, G. Cerullo, A. Leitenstorfer, and R. Huber, "Single-cycle multiterahertz transients with peak fields above $10 \mathrm{MV} / \mathrm{cm}$," Opt. Lett., vol. 35, 26452647,2010

[47] J. Hebling, G. Almási, I. Z. Kozma, and J. Kuhl, "Velocity matching by pulse front tilting for large area THz-pulse generation," Opt. Express, vol. 10, pp. 1161-1166, 2002.

[48] H. Hirori, A. Doi, F. Blanchard, and K. Tanaka, "Single-cycle terahertz pulses with amplitudes exceeding $1 \mathrm{MV} / \mathrm{cm}$ generated by optical rectification in $\mathrm{LiNbO}_{3}$," Appl. Phys. Lett., vol. 98, 091106-1-091106-3, 2011

[49] H. Hamster, A. Sullivan, S. Gordon, W. White, and R. W. Falcone, "Subpicosecond, electromagnetic pulses from intense laser-plasma interaction," Phys. Rev. Lett., vol. 71, pp. 2725-2728, 1993.

[50] D. J. Cook and R. M. Hochstrasser, "Intense terahertz pulses by fourwave rectification in air," Opt. Lett., vol. 25, pp. 1210-1212, 2000.

[51] M. D. Thomson, M. Kreß, T. Löffler, and H. G. Roskos, "Broadband $\mathrm{THz}$ emission from gas plasmas induced by femtosecond optical pulses: From fundamentals to applications," Laser Photon. Rev., vol. 1, pp. 349368, 2007.

[52] M. D. Thomson, V. Blank, and H. G. Roskos, "Terahertz white-light pulses from an air plasma photo-induced by incommensurate two-color optical fields," Opt. Express, vol. 18, pp. 23173- 23182, 2010

[53] K. Y. Kim, A. J. Taylor, J. H. Glownia, and G. Rodriguez, "Coherent control of terahertz supercontinuum generation in ultrafast laser-gas interactions," Nature Photon., vol. 2, pp. 605- 609, 2008.

[54] N. Karpowic, X. Lu, and X.-C. Zhang, "Terahertz gas photonics," J. Mod. Opt., vol. 56, pp. 1137- 1150, 2009

[55] J. Dai, X. Xie, and X.-C. Zhang, "Detection of broadband terahertz waves with a laser-induced plasma in gases," Phys. Rev. Lett., vol. 97, pp. 103903-1-103903-4, 2006.

[56] J. M. Dai, N. Karpowicz, and X.-C, Zhang, "Coherent polarization control of terahertz waves generated from two-color laser-induced gas plasma," Phys. Rev. Lett., vol. 103, pp. 023001-1-023001-4, 2009.

[57] N. Karpowic, J. Dai, X. Lu, Y. Chen, M. Yamaguchi, H. Zhao, X.-C. Zhang, L. Zhang and C. Zhang, M. Price-Gallagher, C. Fletcher, O. Mamer, A. Lesimple, and K. Johnson "Coherent heterodyne timedomain spectrometry covering the entire 'terahertz gap'," Appl. Phys. Lett., vol. 92, pp. 011131-1-011131-3, 2008.

[58] J. Liu, J. Dai, S. L. Chin, and X.-C Zhang, "Broadband terahertz wave remote sensing using coherent manipulation of fluorescence from asymmetrically ionized gases," Nature Photon., vol. 4, pp. 627-631, 2010

[59] C. P. Hauri, C. Ruchert, C. Vicario, and F. Ardana, "Strong-field singlecycle THz pulses generated in an organic crystal," Appl. Phys. Lett., vol. 99, pp. 161116-1-161116-3, 2011.

[60] H. Hirori, M. Nagai, and K. Tanaka, "Excitonic interactions with intense terahertz pulses in $\mathrm{ZnSe} / \mathrm{ZnMgSSe}$ multiple quantum wells," Phys. Rev. B. vol. 81, 081305-1-081305-1(R), 2010.

[61] H. Hirori, K. Shinokita, M. Shirai, S. Tani, Y. Kadoya, and K. Tanaka, "Extraordinary carrier multiplication gated by a picosecond electric field pulse," Nature Commun., vol. 2, pp. 594-1-594-4, 2011.

[62] M. C. Hoffmann, K.-L Yeh, J. Hebling, and K. A. Nelson, "Efficient terahertz generation by optical rectification at $1035 \mathrm{~nm}$," Opt. Express, vol. 15, pp. 11706-11713, 2007.

[63] A. G. Stepanov, J. Kuhl, I. Z. Kozma, E. Riedle, G. Almási, and J. Hebling, "Scaling up the energy of THz pulses created by optical rectification," Opt. Express, vol. 13, pp. 5762-5768, 2005.

[64] M, Jewariya, M, Nagai and K, Tanaka, "Enhancement of terahertz wave generation by cascaded $\chi^{(2)}$ processes in $\mathrm{LiNbO}_{3}$," J. Opt. Soc. Am. B. vol. 26, pp. A101-A106, 2009.

[65] M. Nagai, M. Jewariya, Y. Ichikawa, H. Ohtake, T. Sugiura, Y. Uehara, and $\mathrm{K}$. Tanaka, "Broadband and high power terahertz pulse generation beyond excitation bandwidth limitation via $\chi^{(2)}$ cascaded processes in $\mathrm{LiNbO}_{3}, "$ Opt. Express, vol. 17, pp. 1711543-1711551, 2009. 
[66] J. Hebling, K.-L. Yeh, M. C. Hoffmann, B. Bartal, and K. A. Nelson, "Generation of high-power terahertz pulses by tilted-pulse-front excitation and their application possibilities," J. Opt. Soc. Am. B, vol. 25, pp. B6-B19, 2008.

[67] J. A. Fülöp, L. Pálfalvi, S. Klingebiel, G. Almási, F. Krausz, S. Karsch, and J. Hebling, "Generation of sub-mJ terahertz pulses by optical rectification," Opt. Lett., vol. 37, pp. 557-559, 2012.

[68] M. Nagai, E. Matsubara, and M. Ashida, "High-efficiency terahertz pulse generation via optical rectification by suppressing stimulated Raman scattering process," Opt. Express, vol. 20, pp. 6509-6514, 2012.

[69] J. A. Fülöp, L. Pálfalvi, G. Almási, and J. Hebling, "Design of highenergy terahertz sources based on optical rectification," Opt. Express, vol. 18, pp. 12311-12327, 2010.

[70] M. C. Hoffmann and J. A. Fülöp, "Intense ultrashort terahertz pulses: generation and applications," J. Phys. D: Appl. Phys., vol. 44, pp. 083001-1- 083001-17, 2011.

[71] D. H. Auston, "Subpicosecond electro-optic shock waves," Appl. Phys. Lett. vol. 43, pp. 713-715, 1983.

[72] D. Kleinman and D. Auston, "Theory of electrooptic shock radiation in nonlinear optical media," IEEE J. Quantum Electron., vol. 20, pp. 964970, 1984.

[73] T. Feurer, J. C. Vaughan, and K. A. Nelson, "Spatiotemporal coherent control of lattice vibrational waves," Science, vol. 299, pp. 374-377, 2003.

[74] A. G. Stepanov, J. Hebling, and J. Kuhl, "THz generation via optical rectification with ultrashort laser pulse focused to a line," Appl. Phys. B, vol. 81, pp. 23-26, 2005.

[75] M. Reid and R. Fedosejevs, "Quantitative comparison of terahertz emission from (100) InAs surfaces and a GaAs large-aperture photoconductive switch at high fluences," Appl. Opt., vol. 44, pp. 149153, 2005.

[76] S. H. Autler and C. H. Townes, "Stark effect in rapidly varying fields," Phys. Rev., vol. 100, pp. 703-722, 1955.

[77] A. Mysyrowicz, D. Hulin, A. Antonetti, A. Migus, W. T. Masselink and H. Morkoç, ""Dressed excitons" in a multiple-quantum-well structure: evidence for an optical Stark effect with femtosecond response time," Phys. Rev. Lett., vol. 56, pp. 2748-2751, 1986.

[78] I. I. Rabi, "Space quantization in a gyrating magnetic field," Phys. Rev. vol. 51, pp. 652-654, 1937.

[79] S. E. Harris, J. E. Field, and A. Imamoğlu, "Nonlinear optical processes using electromagnetically induced transparency," Phys. Rev. Lett., vol. 64, pp. 1107-1110, 1990

[80] P. B. Corkum, N. H. Burnett, and F. Brunel, "Above-threshold ionization in the long-wavelength limit," Phys. Rev. Lett., vol. 62, pp. $1259-1262,1989$.

[81] J. L. Krause, K. J. Schafer, and K. C. Kulander, "High-order harmonic generation from atoms and ions in the high intensity regime," Phys. Rev. Lett. vol. 68, pp. 3535-3538, 1992.

[82] P. B. Corkum, "Plasma perspective on strong field multiphoton ionization," Phys. Rev. Lett., vol. 71, pp. 1994-1997, 1993.

[83] M. Y. Ivanov and P. B. Corkum, "Generation of high-order harmonics from inertially confined molecular ions," Phys. Rev. A, vol. 48, pp. 580590, 1993.

[84] S. Ghimire, A. D. DiChiara, E. Sistrunk, P. Agostini, L. F. DiMauro, and D. A. Reis, "Observation of high-order harmonic generation in a bulk crystal," Nature Phys., vol. 7, pp. 138-141, 2011.

[85] B. Zaks, R. B. Liu, and M. S. Sherwin, "Experimental observation of electron-hole recollisions," Nature, vol. 483, pp. 580-583, 2012.

[86] T. Brabec and F. Krausz, "Intense few-cycle laser fields: Frontiers of nonlinear optics," Rev. Mod. Phys., vol. 72, pp. 545-591, 2000.

[87] A. H. Chin, J. M. Bakker, and J. Kono, "Ultrafast electroabsorption at the transition between classical and quantum response," Phys. Rev. Lett., vol. 85, pp. 3293-3296, 2000.

[88] K. B. Nordstrom, K. Johnsen, S. J. Allen, A.-P. Jauho, B. Birnir, J. Kono, T. Noda, H. Akiyama, and H. Sakaki, "Excitonic dynamical FranzKeldysh effect," Phys. Rev. Lett., vol. 81, pp. 457-460, 1998.

[89] A. Srivastava, R. Srivastava, J. Wang, and J. Kono, "Laser-induced above-band-gap transparency in GaAs," Phys. Rev. Lett., vol. 93, pp. 157401-1-157401-4, 2004.

[90] J. R. Danielson, Y. -S. Lee, J. P. Prineas, J. T. Steiner, M. Kira, and S. W. Koch, "Interaction of strong single-cycle terahertz pulses with semiconductor quantum wells," Phys. Rev. Lett., vol. 99, pp. 237401-11237401-4, 2007

[91] S. Schmitt-Rink, D. S. Chemla, W. H. Knox, and D. A. B. Miller, "How fast is excitonic electroabsorption?," Opt. Lett., vol. 15, pp. 60-62, 1990.
[92] S. Hataoka, A. Itoh, I. Tanahashi, and K. Tanaka, "High-density excitation efects of excitons in $\mathrm{ZnSe}$ quantum wells by spectrally resolved four-wave mixing," J. Lumin., vol. 87-89, pp. 853-855, 2000.

[93] S. M. Sze, Physics of Semiconductor Devices. New York: John Willey and Sons, Inc., 1981.

[94] D. K. Ferry, Semiconductor Transport. Lodon: Taylor and Francis, 2000.

[95] A. Leitenstorfer, S. Hunsche, J. Shah, M. C. Nuss, and W. H. Knox, "Femtosecond high-field transport in compound semiconductors," Phys. Rev. B, vol. 61, pp. 16642-16652, 2000.

[96] A. Leitenstorfer, S. Hunsche, J. Shah, M. C. Nuss, and W. H. Knox, "Femtosecond Charge Transport in Polar Semiconductors," Phys. Rev. Lett., vol. 82, pp. 5140-5143, 1999.

[97] W. Kuehn, P. Gaal, K. Reimann, M. Woerner, T. Elsaesser, and R. Hey, "Coherent ballistic motion of electrons in a periodic potential," Phys. Rev. Lett., vol. 104, pp. 146602-1-146602-4, 2010.

[98] J. Hebling, M.C. Hoffmann, H. Y. Hwang, K.-L. Yeh, and K. A. Nelson, "Observation of nonequilibrium carrier distribution in $\mathrm{Ge}, \mathrm{Si}$, and $\mathrm{GaAs}$ by terahertz pump-terahertz probe measurements," Phys. Rev. B, vol. 81, pp. 035201-1-035201-5(R), 2010

[99] L. Razzari, F. H. Su, G. Sharma, F. Blanchard, A. Ayesheshim, H.-C. Bandulet, R. Morandotti, J.-C. Kieffer, T. Ozaki, M. Reid, and F. A. Hegmann, "Nonlinear ultrafast modulation of the optical absorption of intense few-cycle terahertz pulses in n-doped semiconductors," Phys. Rev. B, vol. 79, pp. 193204-1-193204-4, 2009.

[100]F. H. Su, F. Blanchard, G. Sharma, L. Razzari, A. Ayesheshim, T. L. Cocker, L. V. Titova, T. Ozaki, J.-C. Kieffer, R. Morandotti, M. Reid, and F. A. Hegmann, "Terahertz pulse induced intervalley scattering in photoexcited GaAs," Opt. Express, vol. 17, pp. 179620-179628, 2009.

[101]H. Wen, M. Wiczer, and A. M. Lindenberg, "Ultrafast electron cascades in semiconductors driven by intense femtosecond terahertz pulses," Phys. Rev. B, vol. 78, pp. 125203-1-125203-6, 2008

[102]M. C. Hoffmann, J. Hebling, H. Y. Hwang, K. L. Yeh, and K. A. Nelson, "Impact ionization in InSb probed by THz-pump THz-probe spectroscopy," Phys. Rev. B, vol. 79, pp. 161201-1161201-4(R), 2009.

[103]P. Gaal, W. Kuehn, K. Reimann, M. Woerner, T. Elsaesser, and R. Hey, "Internal motions of a quasiparticle governing its ultrafast nonlinear response," Nature, vol. 450, pp. 1210-1213, 2007.

[104]P. A. Wolff, "Theory of electron multiplication in silicon and germanium," Phys. Rev., vol. 95, pp. 1415-1420, 1954.

[105]W. Shockley, "Problems related to p-n junctions in silicon," Solid-State Electron., vol. 2, pp. 35-67, 1961

[106]R. H. Hadfield, "Single-photon detectors for optical quantum information applications," Nature Photon., vol. 3, pp. 696-705, 2009.

[107]J. Chen, V. Perebeinos, M. Freitag, J. Tsang, Q. Fu, J. Liu, and Ph. Avouris, "Bright infrared emission from electrically induced excitons in carbon nanotubes," Science, vol. 310, pp. 1171-1174, 2005.

[108]J. A. Misewich, R. Martel, Ph. Avouris, J. C. Tsang, S. Heinze, and J. Tersoff, "Electrically induced optical emission from a carbon nanotube FET," Science, vol. 300, pp. 783-786, 2003.

[109]B. Tian, X. Zheng, T. J. Kempa, Y. Fang, N. Yu, G. Yu, J. Huang, and C. M. Lieber1, "Coaxial silicon nanowires as solar cells and nanoelectronic power sources," Nature, vol. 449, pp. 885-889, 2007.

[110]R. D. Schaller and V. I. Klimov, "High efficiency carrier multiplication in PbSe nanocrystals: implications for solar energy conversion," Phys. Rev. Lett., vol. 92, pp. 186601-1-186601-4, 2004.

[111]N. M. Gabor, Z. Zhong, K. Bosnick, J. Park, and P. L. McEuen, "Extremely efficient multiple electron-hole pair generation in carbon nanotube photodiodes," Science, vol. 325, pp. 1367-1371, 2009.

[112]J. J. H. Pijpers, R. Ulbricht, K. J. Tielrooij, A. Osherov, Y. Golan, C. Delerue, G. Allan, and M. Bonn, "Assessment of carrier-multiplication efficiency in bulk PbSe and PbS," Nature Phys., vol. 5, pp. 811-814, 2009.

[113]S. Watanabe, N. Minami, and R. Shimano, "Intense terahertz pulse induced exciton generation in carbon nanotubes," Opt. Express, vol. 19, pp. 1528-1538, 2011.

[114]M. L. Cohen and T. K. Bergstresser, "Band structures and pseudopotential form factors for fourteen semiconductors of the diamond and zinc-blende structures," Phys. Rev., vol. 141, pp. 789-796, 1966.

[115]D. A. B. Miller, et al. "Electric field dependence of optical absorption near the band gap of quantum-well structures," Phys. Rev. B, vol. 32, pp. 1043-1060, 1985

[116]R. A. Kaindl, D. Hägele, M. A. Carnahan, and D. S. Chemla, "Transient terahertz spectroscopy of excitons and unbound carriers in quasi-two- 
dimensional electron-hole gases," Phys. Rev. B, vol. 79, pp. 045320-1045320-13, 2009.

[117]C. L. Anderson and C. R. Crowell, "Threshold energies for electron-hole pair production by impact ionization in semiconductors," Phys. Rev. B, vol. 5, pp. 2267-2272, 1972.

[118]M. V. Fischetti, and S. E. Laux, "Monte Carlo analysis of electron transport in small semiconductor devices including band-structure and space-charge effects," Phys. Rev. B, vol. 38, pp. 9721-9745, 1988.

[119]F. Blanchard, D. Golde, F. H. Su, L. Razzari, G. Sharma, R. Morandotti, T. Ozaki, M. Reid, M. Kira, S. W. Koch, and F. A. Hegmann, "Effective mass anisotropy of hot electrons in nonparabolic conduction bands of ndoped InGaAs films using ultrafast terahertz pump-probe techniques," Phys. Rev. Lett., vol. 107, pp. 107401-1-107401-4, 2011.

[120]N. Tzoar and J. I. Gersten, "Theory of electronic band structure in intense laser fields," Phys. Rev. B, vol. 12, pp. 1132-1139, 1975.

[121] Y. Mizumoto, Y. Kayanuma, A. Srivastava, J. Kono, and A. H. Chin, "Dressed-band theory for semiconductors in a high-intensity infrared laser field," Phys. Rev. B, vol. 74, pp. 045216-1-045216-11, 2006.

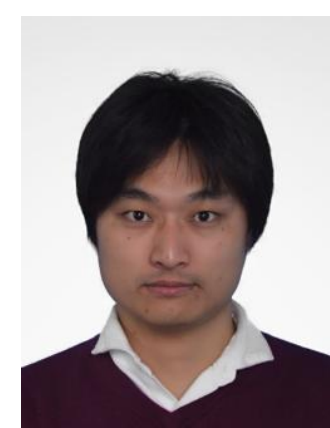

Hideki Hirori received the Ph.D. degree from Kyoto University in 2006. From 2005-2006, he was a Postdoctoral Fellow at the International Research Center for Elements Science Institute for Chemical Research, Kyoto University, Japan. From 2006-2008, he was with Toray Industries, Inc., Japan. In 2008, he joined the Institute for Integrated Cell-Material Sciences (iCeMS), Kyoto, Japan, as a Postdoctoral Fellow, where he is currently an Assistant Professor. His research interests include ultrafast nonlinear optical processes and transport phenomena in nanostructured materials and biological applications of $\mathrm{THz}$ science.

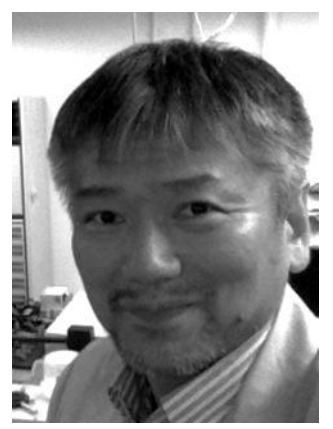

Koichiro Tanaka was born in 1962 at Sapporo and received the B.S. and Ph.D. degrees in physics at Kyoto University, Kyoto, JAPAN in 1985 and 1990, respectively. He was a Research Associate at the institute for Solis State Physics (ISSP) in the University of Tokyo from 1990 to 1995 and joined 'Hirao-Active-Glass Project' at Kyoto (1995 - 1997). In 1997, he moved to the Department of Physics, Kyoto University, JAPAN. Now he is a professor at Institute for Integrated Cell-Material Sciences (iCeMS), Kyoto University. His current research interests include ultrafast spectroscopy of condensed materials and non-linear terahertz spectroscopy of semiconductors and organic compounds. He is a member of Physical Society of Japan, Optical Society of America, and European Optical Society. 